\title{
Bio-Metrological Assessment of Shelf-life of Azadirachta Indica
}

\author{
Balamurugan. R, Sivahari. R, Aravindh. B, Shoba U. S, Sathishkumar. T.
}

\begin{abstract}
The concentration and activity of water in Azadirachta indica describes the shelf life of Neem. The physical and chemical properties of many biological materials depends on the level of water content, which affects the maturity of the fruits and vegetables. Biospeckle technique is a tool used for contrast analysis and hence distinguishes the water activities at different stages in shelf-life of the specimen. Decomposing condition of Azadirachta indica is evaluated by Time History of Speckle Pattern (THSP) during different stages with histogram analysis and fractal box counting methods.
\end{abstract}

Keywords: Azadirachta indica, biospeckle images, histogram, fractal box counting.

\section{INTRODUCTION}

Bio-metrology is a field of science providing a quantitative characterization of biological produce through measurements and data activities could be exploited in order to study the dynamical processes in biological substances and their responses to environment. The optical nondestructive techniques is one of such techniques through which this could be achieved. The adsorption of light by the biological substances introduces changes in the optical path, there by resulting in formation of granular structure called biospeckle, leading to temporal modulations which is a sources of information about activities. Biospeckle technique is an appropriate method to assess the shelf - life period of fruits and vegetables [1-2]. The biospeckle image processing is an inexpensive optical non-destructive technique provides biological activity mapping [3-5]. Self-interference of scattering waves are known as secondary waves from the optically rough surface of the specimen produces bright and dark spot dots. They are called as speckle pattern. The backscattered coherent light from the biological specimen interferes and forms a cigar like speckle images. The speckle pattern becomes steady, if there is no activity inside the sample. Static speckle images from stationary elements of the tissues, whereas the dynamic speckle developing due to the movement or fluctuation of the constituents in the bio sample tissue [6].

Revised Manuscript Received on February 25, 2020.

* Correspondence Author

Balamurugan. R, Department of Physics, Kumaraguru College of Technology, Coimbatore, Tamil Nadu, India. Email: balamurugan.r.sci@kct.ac.in

Sivahari. R* Department of Chemistry, Kumaraguru College of Technology, Coimbatore, Tamil Nadu, India. Email: sivahari.r.sci@kct.ac.in

Aravindh. B, Department of Mechanical Engineering, Kumaraguru College of Technology, Coimbatore, Tamil Nadu, India. Email:aravindh1.18me@kct.ac.in

Shoba. U. S, Department of Chemistry, Kumaraguru College of Technology, Coimbatore, Tamil Nadu, India. Email: ussche@gmail.com

Sathishkumar. T, Department of Biotechnology, Kumaraguru College of Technology, Coimbatore, Tamil Nadu, India. Email: sathishkumar.t.bt@kct.ac.in.

(c) The Authors. Published by Blue Eyes Intelligence Engineering and Sciences Publication (BEIESP). This is an open access article under the CC BY-NC-ND license (http://creativecommons.org/licenses/by-nc-nd/4.0/)

This physical phenomenon of biospeckle or boiling speckle activity explore the shelf life of Azadirachta Indica (AI) as it could reflect the variations of water concentration with respect to time.

Azadirachta indica is a valuable medicinal fruit. The composition, structure and water content decides the ripening conditions of the fruits and it is an important parameter to determine the nutritional values. The

senescence development of fruits relay on post-harvest time and changes in the biological activity. As water constitutes $90 \%$ of the mass level of the sample and its biospeckle activity which relates the degree of wetness or the water activity [7]. Fractal box counting technique is a useful method for quantitative analysis of the bio specimen [8].

In this paper, optical non-destructive biospeckle contrast image analysis on Azadirachta indica was performed to assess the water activity by speckle correlation in different stages with the help of Histogram (with intensity profile) and Time History of Speckle Pattern.

\section{EXPERIMENTAL SETUP}

The laser biospeckle imaging setup consist of Helium Neon laser $(632 \mathrm{~nm}, 1 \mathrm{~mW})$,spatial filter to filter the noise and the beam expander for expanding the laser beam illuminating on the surface of sample with constant intensity of light [9-10] and smooth Gaussian profile can be obtain as output. It is noted that this granulated image developed with time when chemical or bio-physical activity on the illuminated portion occurred [11-13].

Sufficient resolution (1280x1024) charge couple device (CCD) is used to capture the biospeckle images. The sample and the camera distance is adjusted at a distance of about 40 $\mathrm{mm}$ and the laser-sample distance is about $200 \mathrm{~mm}$. The speckle images are captured about 40 degree angle between laser beam and CCD camera, in a regular interval of time period. The experimental setup is shown in Fig (1).

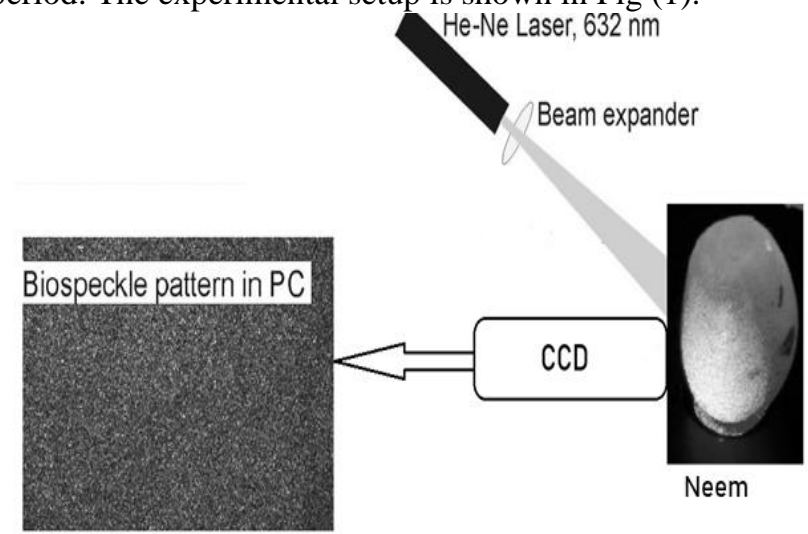

Figure.1. Schematic experimental setup 

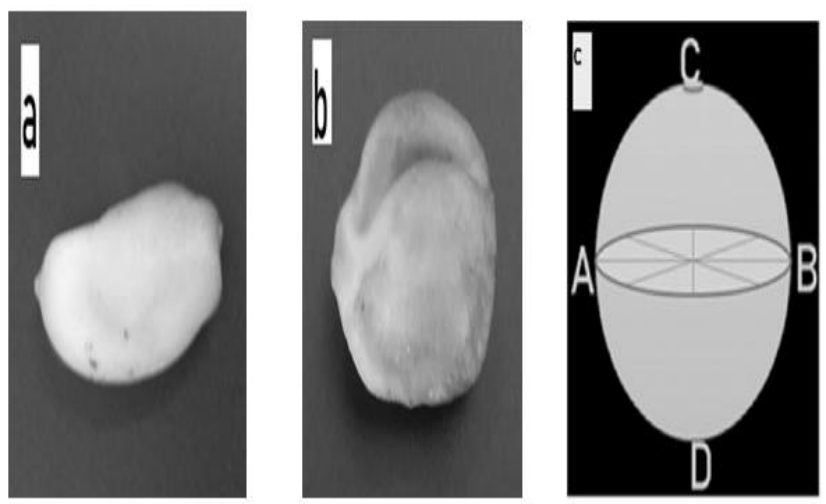

Figure.2. (a) Initial stage (b) last stage of Azadirachta indica (c) Schematic diagram shows the points over the surface of Azadirachta indica

(A, B - equatorial region; $C$ - peduncle insertion and D apex).

\section{RESULTS AND DISCUSSSION}

Fresh Azadirachta indica fruits were taken with approximate size of $3 \mathrm{~cm}$ long and $1 \mathrm{~cm}$ diameter at the center. 512 speckle images were captured by Charge Coupled Device with high resolution. Azadirachta indica dimension variation due to aging effect is shown in Fig. (2). Initial stage of the biospeckle images with the resultant histogram are shown in Fig. (3), Fig. (4). The final stage biospeckle is shown in Fig. (9) with corresponding histogram in Fig. (10).

Dynamic behavior of biospeckle is analyzed by statistical method. Histogram represents the number of pixels in biospeckle image at each different intensity value found in that image. The standard deviation values changes from 29.509 to 47.609 indicating the decrease in water content and the reduction in correlation of the bio sample.

Time History of Speckle Pattern [14] and Co-Occurrences Matrix was created for 512 X 512 composite image. Its THSP rows characterize the dissimilar points on the illuminated sample and represents the intensity of the columns. Speckle time evolution is centered on COM, where $\mathrm{N}_{\mathrm{ij}}$ is the number of occurrences of successive intensity values i,j [15]

$\mathrm{COM}=\mathrm{COM}[\mathrm{Nij}]$

Where, $\mathrm{N}$ is the number of occurrences an assured intensity value $\mathrm{i}$, which is immediately followed by an intensity value $\mathrm{j}$.

Correlation and binary speckle images of the initial stage are depicted in Fig. (5), Fig. (6) and final stage in Fig. (11) \& Fig. (12). Fractal box counting technique is a useful method for quantitative analysis of the bio specimen. Objects which have resemblance and have non-integer scaling dimensions are called fractal. Selection of Fractal box counting is based on the shape and structures of the specimen which is related to the complexity of the object.

Fractal Box Counting of initial stage biospeckle is depicted in Fig. (7) and final stage of Fractal box counting values 2, 4, $6,8,12,16,32,64$ of biospeckle is shown in Fig. (13). Intensity profile, a set of intensity values taken from a regular basis in a lengthwise line segment of initial stage biospeckle is plotted in the Fig. (8) and the final stage in Fig. (14) depicts the reduced water in the sample.

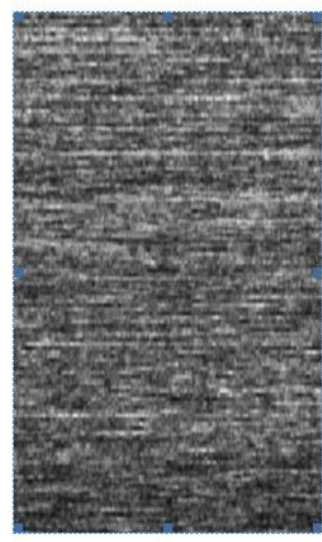

Fig.3. Biospeckle-1.

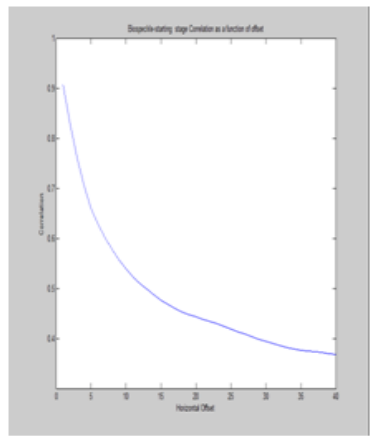

Fig.5. Biospeckle-1 correlation

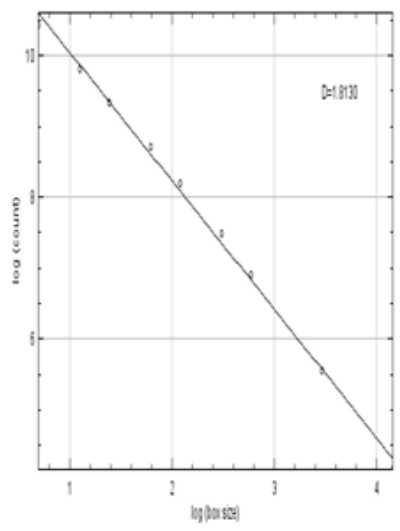

Fig.7. FBC of biospeckle-1

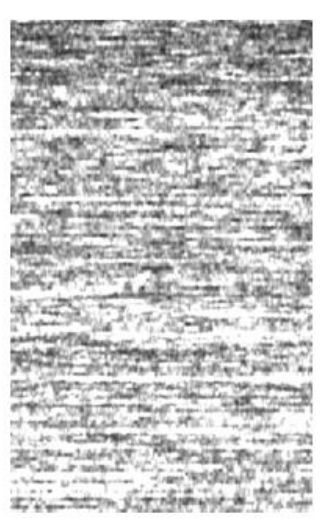

Fig.9.Biospeckle-2.

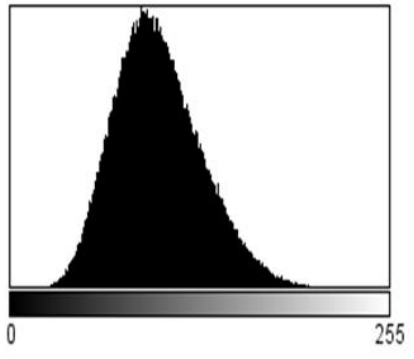

Count 262144 Min: 17 Mean: $99.410 \quad$ Max: 233 StdDev. $29.509 \quad$ Mode: 88 (3761)

Fig.4.Histogram of biospeckle-1.

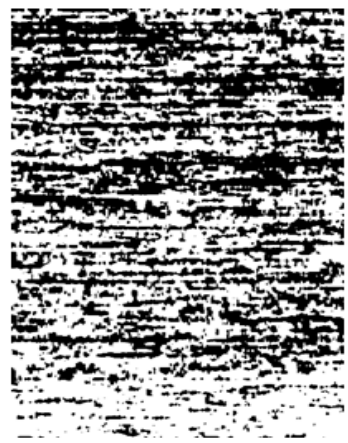

Fig.6.Binary of Biospeckle-1

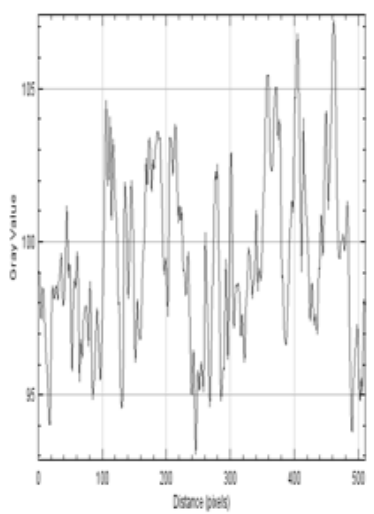

Fig.8. Profile of biospeckle-1

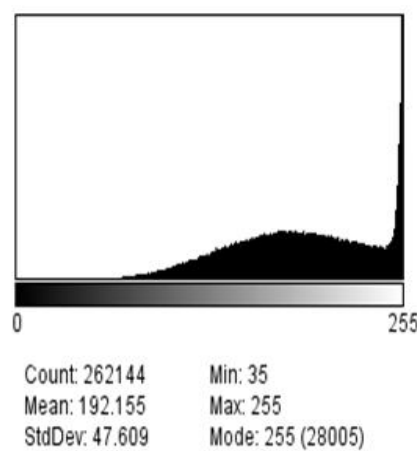

Fig.10. Histogram of biospeckle-2.

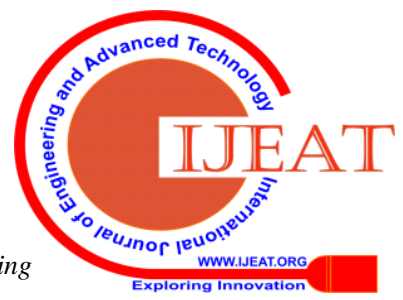




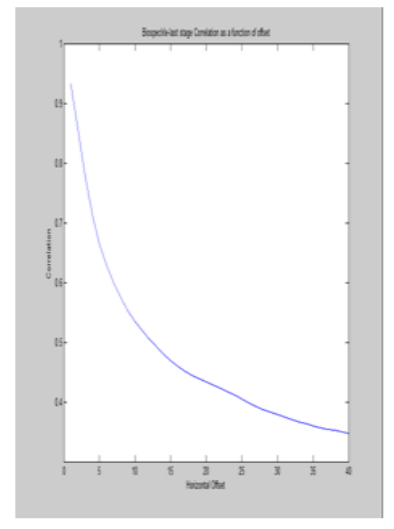

Fig.11. Biospeckle-2 correlation

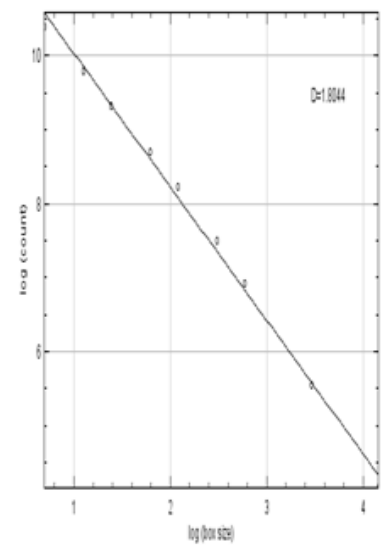

Fig.13. FBC of biospeckle-2

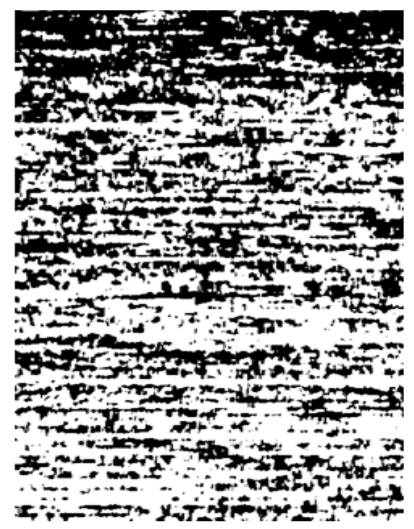

Fig.12.Binary of Biospeckle-2

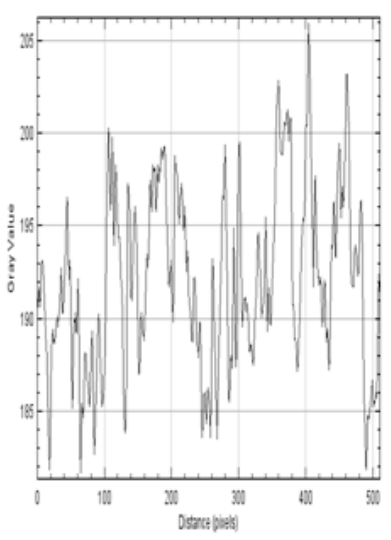

Fig.14. Profile of biospeckle-1
In THSP, change in intensity occurs in the straight direction. Low activity occurs, where the time variations of biospeckle are slow and THSP illustrate the horizontal elongated shape. THSP created from initial stage to final stage shows the higher activity to lower activity on account of the decrease in concentration of water content in the biological specimen.

Co-occurrence matrix of the preliminary state reveals more spread from the diagonal than that of the final state. It means biospeckle of higher activity water content at the initial state shows the inertia moments that gradually decreases to lower activity at the end stage and the corresponding intensity variations. The area of darkness indicates low activity due to dead cells and reduced bio-activity. Among the contrast and concentration of water content correlation linear relationship is noticed.

The water activity declined with increasing concentration due to the presence of water/humidity in the environment. The contrast value indicates the decrease in water activity. Objects inside the specimen having movement causes for the unstable biospeckle pattern. The effects of deterioration of specimen in periodical intervals are observed by the biospeckle activity. The temporal speckle developments are found to increase promptly due to the disturbance in the cellular system and settled down sluggishly with time. Gray level co-occurrence matrix parameters details are given in table-1.
Table-1. GLCM texture features

\begin{tabular}{|l|l|l|}
\hline $\begin{array}{l}\text { Various GLCM } \\
\text { parameters }\end{array}$ & $\begin{array}{l}\text { Initial stage of } \\
\text { biospeckle }\end{array}$ & $\begin{array}{l}\text { Final stage of } \\
\text { biospeckle }\end{array}$ \\
\hline Angular Moment & $4.154 \mathrm{E}-4$ & $5.196 \mathrm{E}-3$ \\
\hline contrast & 71.559 & 234.4 \\
\hline correlation & 0.001 & 0.00041 \\
\hline $\begin{array}{l}\text { Inverse different } \\
\text { Moment }\end{array}$ & 0.177 & 0.1779 \\
\hline Entropy & 8.138 & 8.437 \\
\hline
\end{tabular}

In the initial stage, biological specimen water content is highly active and hence speckle pattern fluctuate due to its fresh condition. Later, due to aging effect, speckle pattern becomes almost smooth due to declining water concentration. Therefore, the correlation of biospeckle images decreases with respect to time. Hence, there is a modification of Inertia Moment swiftly.

Inertia Moment is related to line profiles of the respective THSP images. THSP indicates the concentration of water content and hence its activity changes during the maturity states of the Azadirachta indica. Cross correlation coefficient values decreased rapidly due to concentration of water (aging effect).

\section{CONCLUSIONS}

With the help of laser biospeckle technique, biological and physiological activity of Azadirachta indica was studied. At initial stage, water content of the bio sample was highly active due to its fresh condition and later the correlation of biospeckle images decreases. These variations of contrast in the biospeckle patterns was studied using Fractal Box counting and Histogram methods. Time History of Speckle Pattern illustrated the concentration of water content in Azadirachta indica and its activity changes during the maturity stages.

\section{ACKNOWLEDGMENT}

The authors wish to thank the management of Kumaraguru college of Technology, Coimbatore for the financial support and continuous encouragement for the development of biosensor project work.

\section{REFERENCES}

1. J. A Alves, Braga, R. A. and Vilas Boas, E. V. B. "Identification of respiration rate and water activity change in fresh-cut carrots using biospeckle laser and frequency approach", Postharvest Biology and Technology, 2013, vol. 86, pp. 381-386.

2. R.R Cardoso, A.G. Costa, C.M.B. Nobre and R.A. Braga, "Frequency signature of water activity by biospeckle", laser Opt. Commun. 2011, 284(8), pp. 2131-2136

3. A. Oulamara, G. Tribillon, and J. Duvernoy, "Biological activity measurement on botanical specimen surfaces using a temporal decorrelation effect of laser speckle" J. Mod. Opt., 1989, 36, pp.165-179.

4. 4. M. Szymanska-Chargot, A. Adamiak, A. Zdunek, "Pre-harvest monitoring of apple fruits development with the use of biospeckle method", Sci. Hortic., 2012, 145, pp.23-28. 


\section{Bio-Metrological Assessment of Shelf-life of Azadirachta Indica}

5. K.M. Ribeiro, B. Barreto et al. "Continuous, high-resolution biospeckle imaging reveals a discrete zone of activity at the root apex that esponds to contact with obstacles", Ann. Bot., 2014, 113(3), pp.555-563.

6. I.C. Amaral, J.V. Resende, R.A.et al. "Evaluation of the adsorption behavior of freeze-dried passion fruit pulp with added carriers by traditional biospeckle laser techniques", Dry. Technol 2016, 35, pp.55-65.

7. Sen Men, Lei Yan and Hua Qian, "Application of Bio-speckle Activity to Assess Seed Viability", Advance Journal of Food Science and Technology, 2015, 8(3), pp.214-218

8. R. Balamurugan, R. Prakasam "Shelf-life study of java plum (syzygium cumini) by laser speckle b/d pixel counting technique", Asian Jr. of Microbiol. Biotech. Env. Sc. 2017, Vol. 19, S78-S81.

9. D.A. Boas, A.K. Dunn, (2010) "Laser speckle contrast imaging in biomedical optics", J. Biomed. Opt., 2010, 15, 011109.

10. H.J. Rabal, R.A. Braga, Dynamic Laser Speckle and Applications (CRC Press, Boca Raton, 2008), pp. 282.

11. R. Arizaga, M. Trivi, H. Rabal, "Speckle time evolution characterization by the co-occurrence matrix analysis", Opt. Laser Technol., 1999, 31(2), pp.163-169.

12. R.A. Braga, L. Dupuy, M. Pasqual, R.R. Cardoso, "Live biospeckle laser imaging of root tissues", Eur. Biophys.J., 2009, 38(5), pp.679-686.

13. Arizaga, R, Trivi, M.R., Rabal, H.J. "Speckle time evolution characterization by co-ocurrence matrix analysis", Optics \& Laser Technology, Atlanta, 1999, vol.4-5, n.3, p.1-7.

14. R Balamurugan, G Rajarajan "Study of drying process of paint by dynamic speckle with B/D pixel counting technique", Optics and Lasers in Engineering, 2017, 98, pp.62-68.

15. R.A. Braga, F.P. Rivera., J. Moreira, A Practical Guide to Biospeckle Laser Analysis: Theory and Software (Ed. UFLA, Lavras 2016) p. 158.

\section{AUTHORS PROFILE}

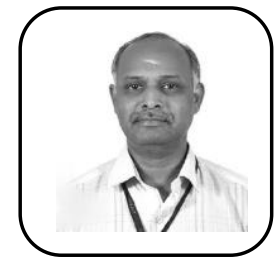

Dr. Balamurugan. R, the author is now working as Faculty of physics in Kumaraguru College of technology, Coimbatore in India and completed two decade of teaching service. He received M.SC. Applied Physics (Instrumentation) from Bharathidasan University, Trichirapalli and M.Phil in Computer science from Manonmanium Sundaranar University, Tamilnadu. He has presented more than fifteen papers in International conferences and published twenty five papers in Laser \& image processing field in Metrology. He has written books in the field of Electronic Devices \& Circuits and Applied Physics. He is a Life member of ISTE and some other science societies/association.

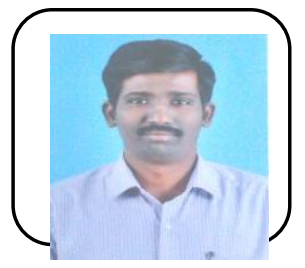

Sivahari. R, faculty in Department of Chemistry with around two decades of proficiency in teaching, adept in handling instruments, is pursuing research in the field chemistry. He has journal publications as well conference presentations to his credit and has authored laboratory manual.

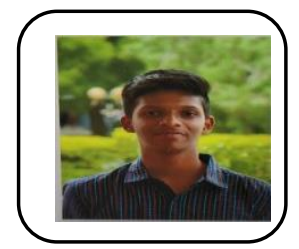

Aravindh. B, the author belongs to mechanical engineering department and interest in optical metrology area. He is an active hardware designer in complex field of experimental work in the domain of opto-mechatranics.

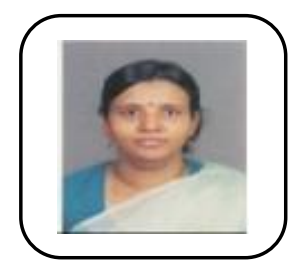

Dr. Shoba U. S. Faculty in department of chemistry, with around two decades of teaching and research experience. Her research initiatives are in Thermoanalytical and Solid-state chemistry, water technology and Algae. She has guided three doctoral degree students and has to her credit few books and journal publications. DST organisations.

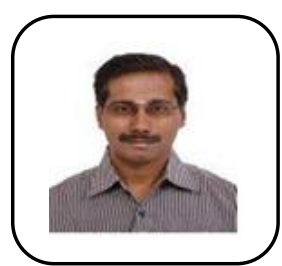

Dr. Sathishkumar.T, the author, working in Department of Biotechnology for more than a decade has vast teaching and research experience. He has published more than 50 journal papers, 3 funded projects and has written 7 books. 